\title{
Die erstaunliche Karriere der Zielvorgabe im Bundesrat
}

\section{Bruno Henggi}

Verantwortlicher Public Affairs, FMH

Der Bundesrat hat ohne vertiefte materielle Diskussion eine fundamentale Weichenstellung für die Gesundheitsversorgung vorgenommen. Die Zielvorgabe für die Kostenentwicklung in der OKP ist zum indirekten Gegenvorschlag der Kostenbremse-Initiative erklärt worden. Der Gesamtbundesrat hat es verpasst, den Plan des Departements des Innern zu durchkreuzen. Das nächste Wort hat das Parlament.

«Der Bundesrat bewegte sich zuletzt in festgefahrenen Bahnen, zeigte eine merkwürdige Unlust am Regieren. Insbesondere Karin Keller-Sutter kritisierte jüngst das ausgeprägte departementale Denken" [1]. Das schrieb die Neue Zürcher Zeitung am 5. Dezember 2018, am Tag, als die Bundesversammlung die Ersatzwahl für den zurückgetretenen Bundesrat Schneider-Amman vornahm.

Die Lust, die interventionistische Politik des Departements des Innern im Gesundheitswesen zu dämpfen, war aber $2018 \mathrm{im}$ Bundesrat durchaus noch vorhanden, als der Berner sein letztes Amtsjahr in der Landesregierung absolvierte. Das Departement des Innern hatte

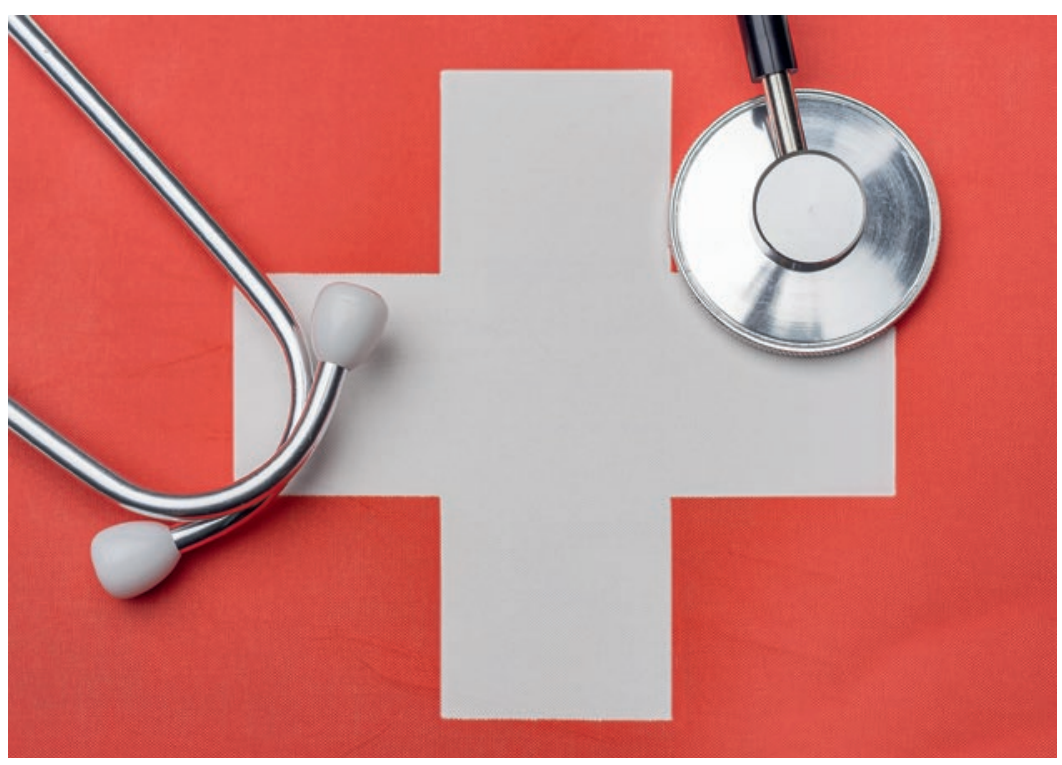

Politische Entscheide beeinflussen die Schweizer Gesundheitsversorgung. geplant, die gesamte Massnahmen-Masse des Berichts der Expertengruppe um Verena Diener, immerhin 38 Massnahmen, dem Parlament in fünf Tranchen vorzulegen. Daraus wurde nichts. An einer Märzsitzung im Jahr 2018 blieben lediglich zwei Massnahmenpakete übrig. Die Parallelimporte für Medikamente, ein Evergreen, blieb auf der Strecke, und die Zielvorgabe für das Kostenwachstum in der OKP, die Prestigemassnahme des EDI, musste auf die Warteliste.

\section{Fokus aufs Departement versus Fokus auf den Bundesrat}

Departementales Denken dominierte damals offensichtlich nicht. Die Mitwirkung einzelner Mitglieder des Bundesrats an den strategischen Entscheidungen des Kollegiums hatte in der Sitzung vom 28. März 2018 sehr wohl stattgefunden. Die Einführung von verbindlichen Zielvorgaben sollte lediglich geprüft werden, und vorerst wollte der Bundesrat nur eine Aussprache führen. Er gab sich dafür Zeit bis Ende 2018. Die Zielvorgabe kam also nicht richtig vom Fleck.

Ein Jahr später trat sie immer noch an Ort. Der Bundesrat will prüfen, und das Departement des Innern erarbeitet eine Vorlage, hiess es am 8. März 2019 offiziell. Weil im Anschluss an eine Sitzung des Bundes mit den Kantonen im Rahmen des «Dialogs Nationale Gesundheitspolitik» das BAG verlauten liess, dass eine Zielvorgabe für die Kostenentwicklung in der obligatorischen Krankenpflegeversicherung Teil der zweiten Vorlage sein werde, verlangte Nationalrat Lorenz Hess in einer Interpellation (19.3798) Auskunft und stellte insbesondere die Frage nach der Prüfung der Verfassungsmässigkeit 
von Zielvorgaben. Erst ein konkreter Regelungsentwurf könne umfassend auf die Verfassungskonformität hin beurteilt werden. Dies werde im Rahmen der Erarbeitung der Vorlage erfolgen, beschied der Bundesrat dem Interpellanten am 21. August 2019.

\section{Fundamentale Massnahme marschiert durch den Bundesrat}

Dann geschah nichts mehr. Die Vorlage verzögerte sich. Der Termin Ende 2019 konnte nicht eingehalten werden. Die neuen Ankündigungen für den Vernehmlassungsbeginn für das zweite Kostendämpfungspaket häuften sich. Im Mai 2020 aber kam der Paukenschlag. "Bundesrat für die Einführung einer Zielvorgabe», entnahm man der Medienmitteilung des Bundesrats. Was war passiert? Bei Lichte betrachtet, nichts Aussergewöhnliches. Eine Initiative war eingereicht worden. Die Volksinitiative für tiefere Prämien - Kostenbremse im Gesundheitswesen der damaligen CVP. Das war alles.

Einen konkreten Regelungsentwurf für die Zielvorgabe gab es nach wie vor nicht. Die Prüfung der Verfassungskonformität fand nicht statt. Trotzdem beschloss die Landesregierung die Einführung einer Zielvorgabe als indirekten Gegenvorschlag zur Kostenbremse. Insider bestätigten, dass es an der Bundesratssitzung zwar Diskussionen gab. Die mit Abstand einschneidendste Massnahme im Kostendämpfungsprogramm des Departements des Innern wurde aber ohne materielle Prüfung Teil des zweiten Kostendämpfungspakets.

\section{Die Einführung von verbindlichen}

Zielvorgaben sollte lediglich geprüft werden, und vorerst wollte der Bundesrat nur eine Aussprache führen.

Eine Aussprache über die Substanz der Zielvorgabe hatte nicht stattgefunden und sollte, wie sich herausstellte, auch später nicht mehr stattfinden. Ein Jahr später, am 28. April dieses Jahres, löste die Landesregierung die Zielvorgabe aus dem zweiten Kostendämpfungspaket heraus. Bis im November soll die entsprechende Botschaft vorliegen.

\section{Tiefe Popularität der Zielvorgabe}

Hätte die Landesregierung Anlass und Grund gehabt, anders zu entscheiden? In der Tat. Desaströs sei das Ergebnis des Vernehmlassungsverfahren gewesen, hiess es in der Bundesverwaltung. 280 Stellungnahmen wurden zur Zielvorgabe eingereicht, eine deutliche Mehrheit lehnt sie ab. Und die Frage der Verfassungs- konformität? Im Vernehmlassungsbericht sind dazu Aussagen enthalten. Allerdings fiel das Kapitel des Bundesamts für Justiz so aus, dass der Sozialversicherungsrechts-Experte Professor Ueli Kieser von der Universität St.Gallen sich veranlasst sah festzustellen, dass die massgebende Fragestellung nicht behandelt worden sei [2].

Die mit Abstand einschneidendste Massnahme im Kostendämpfungsprogramm des Departements des Innern wurde ohne materielle Prüfung Teil des zweiten Kostendämpfungspakets.

Professor Kieser kommt im Gutachten, das er im Auftrag der FMH erstellt hat, zum Schluss, dass die OKP durch eine verfassungsrechtliche Vorgabe als Versicherung konzipiert sei und deshalb nicht über kostendämpfende Massnahmen gesteuert werden könne, welche zum Ziel haben, den Leistungsanspruch zu beschneiden und durch ungenügende Tarifierung dafür zu sorgen, dass Leistungen nicht erbracht werden.

\section{Parlament in der Verantwortung}

Warum also nahm die Karriere der Zielvorgabe seit den Bundesratswahlen Ende 2018 im Bundesrat ihren erstaunlichen Verlauf? Die Staatsrechtslehre mag die Antwort geben: Die Verknüpfung von Kollegial- und Departementalprinzip bewirke, dass jedes Mitglied des Bundesrats eine Doppelaufgabe wahrnehmen müsse. Mitwirkung an den strategischen Entscheidungen im Kollegium und Führung eines Departements. Die erste Aufgabe sei die wichtigere. In der Praxis bestehe aber die Tendenz, dass Bundesräte mehr Gewicht auf die Departementsführung legten, weil ihre Leistungen schwergewichtig daran gemessen würden. Zudem könnten sie sich in dieser Funktion stärker medienwirksam profilieren [3].

Dem Parlament obliegt nun die substantielle Prüfung jener kostendämpfenden Massnahme, welche in Verletzung des Versicherungsprinzips der OKP Leistungsansprüche der Patienten beschneiden und die Erbringung von Leistungen abwenden will.

Bildnachweis

Gregory Alekhin | Dreamstime.com

\footnotetext{
Literatur

1 www.nzz.ch/schweiz/bundesratswahl-auf-einen-blick-ld.1441765

2 www.fmh.ch/politik-medien/politische-geschaefte/kostendaempfung-globalbudget.cfm: Gutachten zur Verfassungsmässigkeit bestimmter Massnahmen im Krankenversicherungsrecht; Prof. Kieser, Seite 28/29.

3 Häfelin U, Haller W, Keller H. Schweizerisches Bundesstaatsrecht. 8. Auflage. Zürich, Basel, Genf; 2012. Seite 528
} 